\title{
Apples, oranges, and lemons: Making sense of lung volume reduction surgery
}

\author{
Joel D. Cooper, MD
}

See related article on pages 2651-8.

The study reported by Decker and colleagues ${ }^{1}$ in this issue of the Journal was undertaken to describe contemporary practice patterns and outcomes of lung volume reduction surgery (LVRS) on a national level, using data obtained from the Society of Thoracic Surgeons (STS) database. The resulting analysis is compared with published results of the National Emphysema Treatment Trial (NETT).

The authors, as have many others, question why application of a procedure demonstrated by the NETT to be so effective for selected patients with emphysema has languished, despite the prevalence of chronic obstructive pulmonary disease.

The STS database was found to contain 538 patients who underwent LVRS over the 8.5-year span covered by the study. However this database is voluntary, so it may or may not be representative of the majority of cases performed in the United States. It contains aggregate deidentified data that does not permit retrospective data mining or subgroup analysis to be conducted. Postoperative data, including morbidity and mortality, is limited to 30 days and does not include either subjective or objective measures of improvement. In comparison with the NETT trial, which included only patients undergoing bilateral LVRS, the STS database cannot distinguish between unilateral and bilateral procedures that have previously been documented to result in differing postoperative mortality and functional outcome. In addition, the limited data designed to be captured by the STS database are often incomplete. For example, 30-day mortality was missing for $14 \%$ of patients so, as the authors indicate, the post-LVRS 30 -day mortality of $5.6 \%$ might actually range from $4.8 \%$ up to $19.6 \%$ depending on how many of the patients with unknown status were actually alive at 30 days. Furthermore the NETT trial demonstrated that postoperative mortality

\footnotetext{
From the University of Pennsylvania Health System, Philadelphia, Pa.

Disclosures: J.D.C. receives a royalty from Synovis Life Technologies for the development of the peri-strip staple line reinforcement used by some surgeons for lung volume reduction surgery.

Received for publication Oct 6, 2014; accepted for publication Oct 8, 2014

Address for reprints: Joel D. Cooper, MD, Division of Thoracic Surgery, Hospital of the University of Pennsylvania, 3400 Spruce St, 6 White, Philadelphia, PA 19104 (E-mail: joel.cooper@uphs.upenn.edu).

J Thorac Cardiovasc Surg 2014;148:2470-1

$0022-5223 / \$ 36.00$

Copyright (c) 2014 by The American Association for Thoracic Surgery

http://dx.doi.org/10.1016/j.jtcvs.2014.10.018
}

following LVRS continues to rise significantly between 30 and 90 days ( $2.2 \%$ and $5.2 \%$, respectively) preventing accurate comparison between the study by Decker and colleagues $^{1}$ and the NETT data. The authors cite a $36 \%$ rate of missing STS data for each of the other outcome variables, and highlight the significant differences in preoperative characteristics between STS database patients and NETT patients. The fact that $34.5 \%$ of patients in the STS database who underwent LVRS are reported as "never smoked" strains credibility. These factors, together with the absence of any postoperative functional data in the STS group emphasize the limitations of this study, all of which are duly noted by the authors.

By attempting to compare STS data with data from the NETT trial, Decker and colleagues ${ }^{1}$ also highlight important limitations and deficiencies of the NETT trial. The NETT trial, a randomized comparison between best medical practice and LVRS, compared outcomes in terms of mortality, exercise capacity, quality of life, pulmonary function studies, and other factors. ${ }^{2}$ Based on information available from prior LVRS reports, the NETT trial had a hypothesis that stated that patients with emphysema who improve following LVRS will preoperatively have "heterogeneously distributed emphysema involving the upper lung zones predominately" (Minutes of NETT Steering Committee, March 24-25 1997). However the actual NETT trial inclusion criteria included many patients who did not meet this hypothesis so as "to include all patients who might benefit." Thus more than $50 \%$ of patients undergoing LVRS in the NETT did not meet the hypothesis criteria. ${ }^{3}$ Furthermore 2 years into the study, disappointing enrollment prompted a change in inclusion criteria, allowing a group of high-risk patients, initially excluded, to be enrolled. A subsequent interim NETT publication reported that LVRS in these patients was associated with a 90day postoperative mortality rate of $30 \%$ and insignificant benefit for those who survived. This group, subsequently referred to as the "high-risk group," was once again reexcluded from the trial.

The definitive NETT report based upon 2-year follow-up data, was published in 2003. This report soon became as controversial as the procedure it was intended to evaluate.

Although demonstrating marked improvement in exercise capacity, health-related quality of life, pulmonary function studies, and others outcome measurements for patients undergoing LVRS, these were considered secondary outcomes and final recommendations were made primarily on the basis of mortality advantage, which was the preselected primary 
outcome measure for the trial. A post-hoc subgroup analysis demonstrated survival benefit at 2 years for a limited number of NETT patients described as having an upper lobe predominant pattern of emphysema and retrospectively classified as having "low baseline exercise capacity." The survival advantage for LVRS in this group was based solely on the early high mortality rate of the corresponding patients randomized to medical therapy.

A later publication by the NETT group 3 years later included $40 \%$ more patients with available 2 -year outcome data and confirmed the 5-year survival advantage of LVRS for the overall group of nonhigh-risk NETT LVRS patients when compared with medical therapy.

A major limitation of the NETT report is the data presentation, which is confusing, highly statistically complicated, and difficult to understand, thus defying the ability to compare it with any other published report on LVRS. The histograms used to compare outcomes between medical and surgical groups do confirm statistical superiority of LVRS over corresponding medical groups, but do not quantify the magnitude of improvement nor allow easy determination of the percent of surviving patients showing improvement at each time point. Furthermore no analysis was presented for the overall subgroup of patients meeting the original NETT hypothesis. It has been noted by others that judging the success or failure only on survival is inadequate and that "every published randomized-controlled trial has shown that LVRS improves symptoms and functioning in survivors." 5

Despite all the misunderstanding and controversy generated by the NETT trial, it was a very comprehensive, wellorganized, and meticulously constructed and conducted trial with strict, well-defined prospectively acquired preand postoperative data. It is a vast database, now available under the Freedom of Information Act, that has been used as a source for many subsequent publications. On the basis of the NETT results, Medicare resumed funding for LVRS.

The scientific conclusions that can be derived from Decker and colleagues' current publication ${ }^{1}$ are quite limited. However the authors are to be congratulated for their overall observations and the recommendations made in their final comments. Underuse of LVRS highlights the paradox that the NETT trial, which has established the benefit achieved by LVRS for appropriate patients, may have significantly limited the very procedure whose value it has confirmed. The pulmonary medicine community has accepted the principle that reduction of hyperinflation in selected patients with chronic obstructive pulmonary disease can produce significant functional, quality of life, and survival benefit as judged by the multiple attempts to achieve similar benefit by a variety of endoscopic procedures. Yet none of these has demonstrated sufficient effectiveness to warrant approval in the United States while referrals for LVRS are few and far between.

There have been more than enough experience, publications, and clinical trials of LVRS to analyze, clarify, and define its appropriate role, risks, and benefits. Decker and colleagues ${ }^{1}$ suggest what is now needed is consensus on how to ensure that LVRS is appropriately used and the importance of developing multidisciplinary centers for the treatment of advanced respiratory disease for both surgical and medical management of emphysema. This publication, although confirming the difficulty in comparing apples and oranges, may by its recommendations produce an improved product, confirming yet again the adage that when confronted with lemons, the best thing to do is make lemonade.

\section{References}

1. Decker MR, Leverson GE, Jaoude WA, Maloney JD. Lung volume reduction surgery since the National Emphysema Treatment Trial: Study of Society of Thoracic Surgeons Database. J Thorac Cardiovasc Surg. 2014;148:2651-8.

2. National Emphysema Treatment Trial Research Group. A randomized trial comparing lung-volume-reduction surgery with medical therapy for severe emphysema. N Engl J Med. 2003;348:2059-73.

3. Sanchez PG, Kucharczuk JC, Su S, Kaiser LR, Cooper JD. National emphysema treatment trial redux: accentuating the positive. J Thorac Cardiovasc Surg. 2010; 140:564-72.

4. Naunheim KS, Wood DE, Mohsenifar Z, Sternberg AL, Criner GJ, DeCamp MM et al. Long-term follow-up of patients receiving lung-volume reduction surgery versus medical therapy for severe emphysema by the national emphysema treatment trial research group. Ann Thorac Surg. 2006;82:431-43.

5. Yusen RD, Littenberg B. Integrated survival and quality of life data in clinical trials of lung disease: the case of lung volume reduction surgery. Chest. 2005; 127:1094-6. 